\title{
ADA clinical recommendations on topical fluoride for caries prevention
}

\author{
Abstracted from \\ Weyant RJ, Tracy SL, Anselmo T et al. \\ Topical Fluoride for Caries Prevention; Full report of the updated clinical recommendations and supporting systematic review. American Dental \\ Association 2013 \\ Address for correspondence: Dr Tracy, Centre for Evidence-Based Dentistry, Division of Science, American Dental Association, Chicago, IL, USA
}

Scope and purpose This guideline is intended to assist practitioners with decision making about the use of topical fluoride caries-preventive agents. It presents evidence-based clinical recommendations on professionally applied and prescription strength, home-use topical fluoride agents for caries prevention. Three clinical questions on the use of topical fluoride caries-preventive agents are addressed:

- In primary and permanent teeth, does the use of a topical fluoride compared to no topical fluoride reduce the incidence of new lesions, or arrest or reverse existing coronal and/or root caries?

- For primary and permanent teeth, is one topical fluoride agent more effective than another in reducing the incidence of, or arresting or reversing coronal and/or root caries?

- Does the use of prophylaxis before application of topical fluoride reduce the incidence of caries to a greater extent than topical fluoride application without prophylaxis?

Sodium, stannous and acidulated phosphate fluoride for professional and prescription home use, including varnishes, gels, foams, rinses and prophylaxis pastes were evaluated.

Methodology The previous version of this review (2006 ADA policy) was based on assimilation of evidence from systematic reviews. However, this update synthesises primary evidence collected through systematic review and appraisal of the literature.

The Cochrane Library and Medline (via PubMed) were searched. Relevant systematic reviews and other selected articles were handsearched.

Two reviewers independently screened titles and abstracts, with one reviewer assessing all manuscripts in full for meeting the inclusion criteria. Two different members of the expert panel then approved the exclusion list. Discrepancies were resolved through discussion with the Chair of the workgroup. The USPSTF grading system was used. Review and updating Five-year interval update in line with the ADA policy.

Recommendations For individuals at risk of dental caries: $2.26 \%$ fluoride varnish or $1.23 \%$ fluoride (APF) gel, or prescription strength, home-use $0.5 \%$ fluoride gel or paste, or $0.09 \%$ fluoride mouth rinse for children who are aged six or over.

The panel judged that the benefits outweighed the potential for harm for all professionally applied and prescription strength, home-use topical fluoride agents and age groups except for children aged under six years. Only $2.26 \%$ fluoride varnish was recommended for children less than six years old, as the panel considered the risk of adverse events (particularly nausea and vomiting) associated with swallowing all other professionally applied topical fluoride agents outweighed the potential benefits.

There was insufficient data to answer the question on arresting and reversing coronal and/or root caries, so these outcomes were not addressed in the clinical recommendations. Research recommendations These focused on development of standard methodologies for well-designed trials with standardised reporting and trial registration. Specific areas for research included: investigation of mechanisms of fluoride action and effects against a background fluoride exposure; investigation of specific sub-groups such as high-risk elderly; research on specific products; measure and outcome development for arrested caries; economics and dissemination/implementation to realise guideline use.

\section{Commentary}

These updated guidelines replace the 2006 guidelines for professionally applied topical fluoride (PATF) use, which were limited to $\mathrm{F}$ varnish and $\mathrm{F}$ gels for all individuals and for individuals aged six or older respectively, who were at higher risk of caries. ${ }^{1}$

The report had two main aims: first, to provide an update on the efficacy of PATF use based on a thorough review of the clinical evidence, and to determine the need for prophylaxis prior to application of topical fluorides. The systematic review concluded that topical fluorides do provide additional benefit, but that prophylaxis prior to their use did not improve efficacy compared with no prophylaxis. Second, the panel sought to address additional questions related to prescription strength home-use topical fluorides for caries prevention.

It is this introduction of 'prescription level' concentrations of topical fluorides for home use from the age of six years where these US guidelines differ fairly substantially from international equivalents. However, the reporting of the guidelines are a little unclear; the overall guidance summary table appears to provide two alternatives for office use 'AND' two alternatives for home use while the report's abstract, through the extensive use of the word ' $O R^{\prime}$, 
appears to advocate the use of ONLY ONE topical fluoride, with the decision on its form and location of use being made by the dentist, using their professional judgement and having considered their patient's needs and preferences.

These new guidelines reiterate that the recommended topical fluoride use is in addition to drinking fluoridated water and using over the counter (OTC) strength fluoridated toothpastes, recommended for use by all individuals, irrespective of caries risk. However, the decision to widen the use of a $0.09 \% \mathrm{~F}$ (900 ppmF) mouthrinse from its previous main area of use, for example weekly supervised mouthrinsing in schools, is a major change. This product is recommended for 'at least weekly use' in the home arena in individuals older than six years, with a $0.5 \%$ (5000ppmF) fluoride gel or paste as an alternative measure, also for home use in the same age groups.

The evidence the panel considered was sufficient to allow the designation of 'in favour' for mouthrinse use in six to 18-year olds, while for home-based mouthrinse use in older individuals, homebased use of $\mathrm{F}$ gel/pastes, and professionally applied $\mathrm{F}$ varnishes and APF gels the strength of the recommendation was only at the level of 'expert opinion for' its use. The limitations to the existing literature on topical fluorides for caries prevention were clearly highlighted by the panel who concluded that further clinical research was necessary using standardised methods and reporting.

Considering the impact of these guidelines on the various stakeholders, the need to monitor systemic fluoride exposure in vulnerable groups; for example six-nine-year olds, will be enhanced as inadvertent ingestion of fluoride may increase through exposure to additional sources at home. It is clear that there is not yet universal compliance with responsible supervision and use of toothpastes in younger children and the ADA have also recently reinforced their advice in this area. ${ }^{2}$

\section{Practice points}

- It is important for prescribing dentists to carefully assess which families would benefit from home based higher $F$ concentration topical fluoride use and which might be best managed in traditional practice-based PATF programmes.

- Ensuring parental/carer responsibility in strictly following manufacturers' instructions for use when supervising the dispensing and use of $\mathrm{F}$ containing products in recipient children is an important professional challenge.

Anne Maguire

Centre for Oral Health Research, School of Dental Sciences, Newcastle University, Framlington Place, Newcastle upon Tyne, UK

1. Professionally applied topical fluoride: evidence-based clinical recommendations. I Am Dent Assoc 2006; 137: 1151-1159.

2. Fluoride toothpaste use for young children. J Am Dent Assoc 2014; 145: 190-191.

Evidence-Based Dentistry (2014) 15, 38-39. doi: 10.1038/sj.ebd.6401019 